\title{
Primary cutaneous diffuse large B-cell lymphoma, leg type - case report and literature review
}

\author{
Dorina Nastasia PETRA ${ }^{1,2}$, Andreea VARGA ${ }^{1,2}$, Smaranda DEMIAN $^{1,3}$, \\ Emoke HORVATH ${ }^{1,4}$, Ioan TILEA ${ }^{1,2}$ \\ 1"George Emil Palade" University of Medicine, Pharmacy, Science and Technology, \\ Targu Mures, Romania \\ 2Internal Medicine II Clinic, Emergency Clinical County Hospital, Targu Mures, Romania \\ ${ }^{3}$ Internal Medicine I Clinic, Emergency Clinical County Hospital, Targu Mures, Romania \\ ${ }^{4}$ Pathology Department, Emergency Clinical County Hospital, Targu Mures, Romania
}

\begin{abstract}
The primary cutaneous diffuse large B-cell lymphoma leg type (PCDLBCL-LT) is a rare form of cutaneous lymphoma with aggressive and unpredictable evolution. The patients are often aged and with comorbid conditions so that immunochemotherapy can be poorly tolerated. Despite its complexity, a differential diagnosis is possible in the primary care setting and it is of great importance in guiding further investigation and referral. We present the case of a 70-year-old man with reddish nodular lesions appeared on the anterior region of the right tibia. Anatomopathological subtype identified a PCDLBCL-LT, T2aN1M0 stage. The first line of R-CHOP regimen (rituximab - cyclophosphamide, doxorubicin, vincristine, prednisone) associating intrathecal prophylaxis with methotrexate was prescribed with a good response. An early relapse after first line chemotherapy was noticed. Salvage R-DHAP regimen (rituximab-dexamethasone, high dose cytarabine, cisplatin) with modest response and progression of the disease under chemotherapy was instituted. During targeted therapy, the patient experienced an acute myocardial infarction, heart failure, and acute kidney injury. Thus, comorbid conditions have dramatically reduced systemic therapeutic options. The multidisciplinary approach with the implication of general physician, hematologist, cardiologist and other specialists was necessary for the management of this case.
\end{abstract}

Keywords: primary cutaneous diffuse large B-cell lymphoma, leg type, diagnosis, treatment, relapse, multidisciplinary approach

\section{INTRODUCTION}

In the primary care setting skin-related changes are commonly encountered. Family physicians are responsible for a thorough history and clinical examination of the patient, along with the evaluation of the skin le- sions and a detailed description of their features in order to establish which lesions may warrant further referral. A comprehensive approach narrows the diagnostic work-up and guides further exams. Primary cutaneous lymphomas (PCLs) are rare, extranodal non-Hodgkin`s lymphomas with the second incidence 
after gastrointestinal lymphomas (1). Primary cutaneous B-cell lymphomas (PCBCL) represents a minority in the group of PCLs (around 20-25\%), the majority being T-cell lymphomas $(2,3,4)$. European Organization for Research and Treatment of Cancer, EORTC2, revised 2017 and 2018 (3) World Health Organization WHO Classification of the mature B-cell, T-cell, and NK-cell neoplasms recognise three main types of PCBCLs, two with indolent course and over $90 \%$ five years overall survival (primary cutaneous marginal zone lymphoma and primary cutaneous follicle-center lymphoma) and one with aggressive behaviour, frequent relapses and significantly poorer survival rates, primary cutaneous diffuse large B-cell lymphoma, leg type (PCDLBCL-LT) (1-7).

PCDLBCL-LT is a sporadic disease, with a frequency of around $10-20 \%$ of PCBCLs (3-6) and $4 \%$ of all PCLs (2). Tumours arise mainly on the lower limbs; relapse rate is high, and extracutaneous extensions (lymph nodes or even central nervous system) are reported $(5,6,8)$. The primary care physician may be able to formulate a presumptive diagnosis based on the available information regarding the clinical features. For an adequate initial work-up history, complete physical examination, laboratory tests (complete blood count and chemistry), bone marrow biopsy, computed tomography or fluorodeoxyglucose positron emission tomography are highly recommended $(1,2,3)$. Diagnosis is based on the proper skin biopsy evaluation, with accurate morphology and immunohistochemistry (IHC) (1). A particular TNM (tumor, node, metastases) system (9) is used for staging. The treatment is similar to another lymphoma non-germinal center B-cell - type diffuse large B-cell (non-GCB DLBCL): systemic chemotherapy +/- involved fields radiation $(1,3)$.

The main objective of this paper is the presentation of a rare and aggressive non-Hodgkin's lymphoma, PCDLBCL-LT, that required a multidisciplinary approach.

\section{CASE PRESENTATION}

A 70-year old Caucasian male was referred to a family medicine practice the first time in March 2017, presenting with reddish nodular lesions appearing in the anterior region of the right tibia, infiltrating the tissues with local edema (figure 1) and multiple small lymph nodes in the right inguinal region.

The initial lesion occurred six months ago and gradually increased in size, more accelerated in the last month. The patient had no constitutional B symptoms (sweating, fever or weight loss) and had a good ECOG (10) performance status (ECOG-1). Family medical history does not reveal significant features and controlled hypertension, mitral valve regurgitation and benign prostatic hyperplasia were noted in personal medical history.

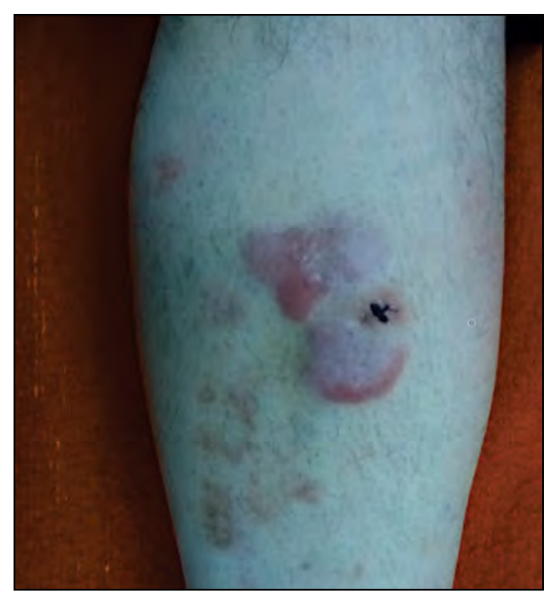

FIGURE 1. Clinical aspect of the right leg at first presentation

Initial laboratory findings were within normal limits excepting a high lactate dehydrogenase level. The tumor biopsy revealed a diffuse lymphoid infiltrate with atypical cells, large nuclei, crunchy chromate, nucleoli, and cytoplasm in varying amounts, Ki67 proliferation index between $70 \%$ and $80 \%$. Immunohistochemical analysis identified neoplastic cells positive for CD20, Pax5, and BCL-2 and with no expression of Cyclin D1, CD3, CD10, CD23 or BCL-6 (figure 2).

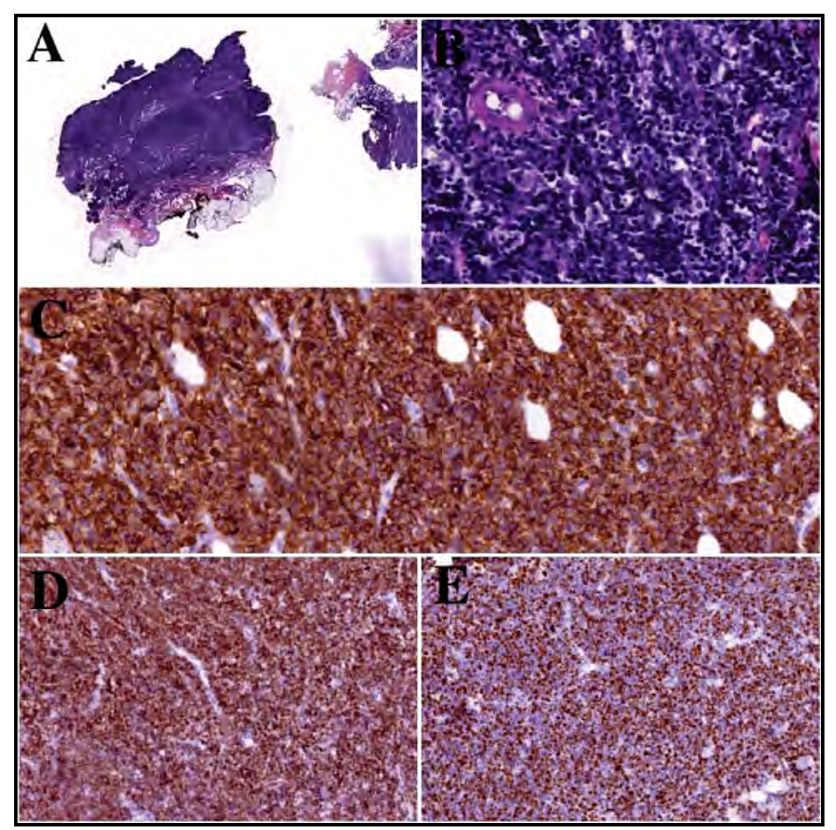

FIGURE 2. Immunohistochemical analysis of a fragment obtained from biopsy specimen

FROM UPPER LEFT: A. Primary cutaneous DLBCL, leg type: tumour cells in the cutaneous tissue forming compact tumour mass (HE staining, $1 X$ )

B. The tumour is comprised of a monotonous diffuse infiltrate with large cells, showing prominent nucleoli (HE, 4OX)

C. The neoplastic cells express CD20 (DAB chromogen, 20X)

$D$. The neoplastic cells express BCL-2 (DAB chromogen, 20X) $E$. The proliferation fraction detected by Ki67 is between 70 80\% (DAB chromogen, 20X) 
Bone marrow biopsy does not detect any tumor infiltration. Full-body computed tomography does not reveal lymphadenopathy or other pathological changes, but ultrasound exam indicated the presence of some small lymphadenopathy in the right inguinal region. Magnetic resonance imaging study revealed at the level of upper and middle thirds of the right shank a mass with tissue signal, active in diffusion, homogeneous gadolinophylia, with expansion in the soft parts (muscle and fat). The final diagnosis was PCDLBCL-LT, stage T2aN1M0.

The first-line chemotherapy applied was R-CHOP regimen (rituximab-cyclophosphamide, doxorubicin, vincristine, prednisone) six courses, with intrathecal methotrexate prophylaxis, considering the increased risk of dissemination in the central nervous system due to the histological subtype itself, increased lactate dehydrogenase value and advanced age. A proper initial response was described, with tumor dissolution, thus consolidative involve-sites radiation therapy was planned.

Unfortunately, a very early disease relapses occurred (less than three months) after the completion of chemotherapy. Multiple, rapidly growing nodules, at the level of the right tibia and the scalp, appeared. At this time, the patient displays evident B symptoms and an impaired ECOG performance status (ECOG-3). The laboratory revealed secondary thrombocytosis $(549,000 / \mathrm{mmc})$ and high-level lactate dehydrogenase (357 U/I). Biopsy of the lesions was repeated with the similar results. Bone marrow biopsy was again without any signs of tumoral infiltration. Computer tomography for restaging revealed highlights iodophil nodules, placed extracranial, bilateral occipital and in the anterior region of the right tibia, with a maximum diameter of $23 \mathrm{~mm}$.

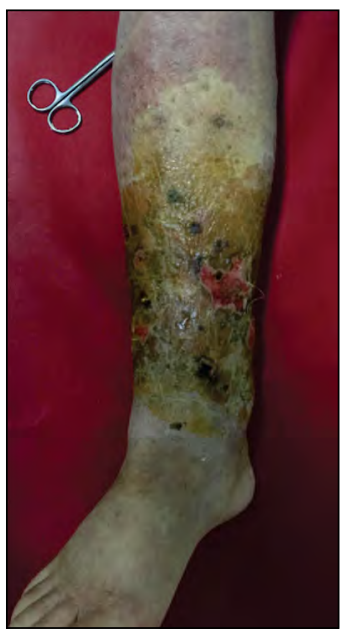

FIGURE 3. Clinical aspect of the right leg

Salvage R-DHAP regimen (rituximab-dexamethasone, high dose cytarabine, cisplatin) was started, with a prophylactic granulocyte growth factor. Due espe- cially to age but also to comorbidities and the personal option, the patient does not qualify as a viable candidate for autologous stem-cell transplantation. After the first R-DHAP cycle the tumoral formations regressed but shortly the neoplasm begins to progress. The tumours at the level of the right leg reappeared with a rapid growth rate, associating deep vein thrombosis. More nodules, with subsequent ulceration, continued to appear on the anterior thorax, and contralateral inferior limb (figure 3).

As a consequence of the personal risk factors, comorbidities, and chemotherapy - related toxicity the patient developed acute myocardial infarction, significant heart failure (NT-proBNP: $8,425 \mathrm{pg} / \mathrm{ml}$ ) and acute kidney injury (eGFR: $26 \mathrm{ml} / \mathrm{min} / 1.73 \mathrm{~m}^{2}$ - CKD-EPI formula). Echocardiography described hypokinesia of the apex and apical third of the anterior wall, a moderate depressed left ventricular ejection fraction (LVEF: $42 \%)$, mild mitral regurgitation and diastolic dysfunction, altered relaxation type. At that time we concluded that a coronary angiogram was inappropriate due to the clinical status. Thus, the chemotherapeutic options were significantly limited; anthracyclines could not be administered due to severe myocardial injury. Despite multifaceted treatment, the patient died 20 months after diagnosis, due to neoplasia itself and cardiovascular complications.

\section{DISCUSSIONS}

PCDLBCL-LT is a rare entity. PCDLBCL-LT affects elderly patients, with a median age at diagnosis over 70 year (4-9). Female are more involved, but not in all studies $(5-8,11,12)$. Particular risk or causing factors are not well defined, but immunosuppression or oncogenic viral infection may play a role $(13,14)$.

The clinical examination identified reddish or plum-coloured solitary or multiple, fast-growing nodule-shape tumours or plaques; main distribution is the lower limb, uni or bilaterally, especially below the knee (4-9). Some less usual clinical presentations were reported, mimicking inflammatory dermatoses or even a chronic venous ulcer $(13,14,15)$. Less frequently, the disease may also develop or spread at other cutaneous (trunk, head-neck, upper limb) $(4-8,11)$ or extracutaneous sites like lymph nodes, bone marrow or even the central nervous system $(4-9,11,12,15)$.

Massone $C$ et al. (16) reported in 2015 a series of atypical PCDLBCL-LT presenting as asymptomatic, erythematous annular patches and thin plaques, in contrast with the more commonly seen thick plaques or tumours. The differential diagnosis included inflammatory dermatoses, such as granuloma annulare characterized by the development of papules in an annular arrangement (17), necrobiosis lipoidica, sarcoidosis, 
erythema annulare centrifugum and erythema marginatum. One of the cases was interpreted by the general practitioner as an erythema chronicum migrans lesion caused by Borrelia burgdorferi and was treated accordingly. The lack of response to antibiotic regimen lead to further investigation, and obtaining of a biopsy specimen that guided the definitive diagnosis. In rare cases, large, ulcerated lesions may be the clinical presentation of PCDLBCL-LT, misleading to a chronic venous ulcer diagnosis $(18,19)$. A non-healing leg ulcer should invite to a reconsideration of diagnosis by the primary care physician.

In its classical presentation form, PCDLBCL-LT can be easily misinterpreted. A first approach should take into consideration the differential diagnosis of conditions presenting as soft tissue masses, with a plethora of causes ranging from infection to trauma (20). Age, history, and clinical manifestations of the patient, along with the examination of the mass with regards to its aspect and anatomic location can aid in narrowing the diagnosis. The clinical appearance of the presented case, at the diagnosis, was a typical one. The patient presented initially with a solitary tumor on the right leg without any extracutaneous dissemination, excepting a possible (but not histologically proved) invasion of same-side inguinal lymph nodes. At the disease progression time, tumours appeared not just on the leg but at distance (on the scalp) too, again no clear extracutaneous dissemination was detected. TNM9 stage was T2aN1M0 at diagnosis and T3aNOMO at the relapse time.

For an accurate definite and differential diagnosis of PCDLBCL-LT, the proper skin biopsy is mandatory. The morphology appearance is typically a diffuse infiltration of the full dermis (often the subcutis too and sparing the epidermis) with centroblasts and immunoblasts, without admixed small lymphocytes (6$8,11,12)$. Sometimes morphology can be a challenging and confusing one $(21,22)$.

Immunohistochemistry (IHC) reveals positivity of pan B-cell markers, BCL-2 positivity is common (85$90 \%)$, BCL-6 may be positive (45-75\%) and CD10 is generally negative (positivity under $6 \%)(6,11,12)$. Other IHC markers with frequent positive staining are MUM-1, FOX-P1, IgM, and p63 $(6,11,12,21-24)$. In particular cytoplasmic IgM appears as a sensitive marker of PCDLBCL-LT $(23,24)$. Other IHC characteristic of PCDLBCL-LT is the negativity of CD5, CD10, CD30, $\mathrm{CD} 138$, and Cyclin D1, as valuable tools for the differential diagnosis from other PCBCL $(6-8,11-13,21,22)$. The BCL-2 expression seemed to have a prognostic impact in some studies (22) but was not demonstrated in others $(6,11)$. Proliferation index Ki67 is high, around $70 \%(40-90 \%)$ (22). In the above presented case IHC stained positive in malignant cells for CD20, PAX-5, and
$\mathrm{BCL}-2$, and negative for CD23, CD3, CD5, CD10, BCL-6 with a Ki67 index between 70 and $80 \%$.

Molecular and genetic studies revealed for PCDLB$\mathrm{CL}-\mathrm{LT}$ a phenotype and gene expression similar to primary nodal non-GCB DLBCL or primary central nervous system DLBCL (21,25-28). Constitutive activation of nuclear kappa light chain enhancer of activated B-cells (NF-KappaB), mutations of myeloid differentiation primary response gene (MYD88) especially L265P variant, mutations in the cluster of differentiation $79 \mathrm{~B}$ (CD79B), mutations of transducin beta-like 1X-linked receptor 1 (TBL1XR1) gene was found to have a high frequency (25-30). MYD88 L265P mutation is proposed as a genetic marker of PCDLBCL-LT $(26,28)$ and appears as a valuable argument for the use of ibrutin$i b$, a selective inhibitor of Bruton tyrosine kinase targeting the MYD88 pathway, in the treatment of cases who fails conventional therapy (26-32).

PCDLBCL-LT has an aggressive behaviour, a significantly poorer prognosis than other $\mathrm{PCBCL}$, with overall survival at five years around $50 \%$ and common recurrences $(1-9,12,13,21,22,33)$. Treatment recommendations are based merely on relatively small studies or expert experience, because large, prospective, controlled studies are missing $(1,3,12)$. Management is similar to a high-risk, limited stage, systemic non-GCB $\operatorname{DLBCL}(1,3,12)$. R-CHOP immunochemotherapy +/- involved sites radiation is used at first line; for the relapsed disease international guidelines recommend salvage therapy, palliative radiotherapy or ibritumomab tiourexan $(1,3)$. Ibrutinib or lenalidomide has been used off-label in some cases, with good results reported $(31,34)$. There are also sporadic cases of PCDLBCL-LT presented, with apparently spontaneous tumor regression (but not complete remission) and T-cell massive infiltration of involved sites $(35,36)$. Rao $S$ et al. propose an interesting palliative local treatment, intralesional gentian violet (37).

Our PCDLBCL-LT case responded initially well to first-line therapy, but progression was early and aggressive. The patient did not well-tolerate the non-haematological therapy (for coronary artery disease, heart failure and kidney injury) and the lymphoma appeared as a chemoresistant one.

Despite its complexity, a differential diagnosis of primary cutaneous diffuse large B-cell lymphoma it's possible in the primary care setting and it is of great importance in guiding further investigations and referrals.

\section{CONCLUSIONS}

PCDLBCL-LT is a rare entity with a challenging diagnostic work-up and treatment. The disease is an aggressive one and patients are often older so that im- 
munochemotherapy is expected to be poorly tolerated. That was the case for the presented patient too; comorbid conditions (myocardial infarction, heart failure, kidney disease) dramatically reduced systemic therapeutic options. Nevertheless, a definitive diagno- sis and the management of such cases require a multidisciplinary approach with the implication of general physician, hematologist, dermatologist and other specialists.
Conflict of interest: none declared Financial support: none declared

\section{REFERENCES}

1. Willemze R, Hodak E, Zinzani PL et al. Primary cutaneous lymphomas: ESMO clinical practice guidelines for diagnosis, treatment and follow-up. Ann Oncol. 2018;29(Supp 4):iv30-iv40.

2. Willemze R, Jaffe ES, Gunter B et al. WHOEORTC classification for cutaneous lymphomas. Blood. 2005;105(10):37683785.

3. National Comprehensive Cancer Network NCCN. Clinical practice guidelines in oncology (NCCN Guidelines) primary cutaneous B-cell lymphomas, Version 2.2018-January 10. 2018 - https://www. nccn.org/professionals/physician_gls/pdf/ pcbcl.pdf.

4. Bradford PT, Devesa SS, Anderson WF et al. Cutaneous lymphoma incidence patterns in the United States: a population-based study of 3884 cases. Blood. 2009;113(21):5064-5073.

5. Zinzani PL, Quaglino P, Pimpinelli $N$ et al. Prognostic factors in primary cutaneous B-cell lymphoma: the italian study group for cutaneous lymphomas. J Clin Oncol. 2006;24(9):1376-1382.

6. Senff NJ, Hoefnagel JJ, Jansen PM et al. Reclassification of 300 primary cutaneous B-cell lymphomas according to the New WHO-EORTC classification for cutaneous lymphomas: Comparison with previous classifications and identification of prognostic markers. J Clin Oncol. 2007;25(12):1581-1587.

7. Lima M. Cutaneous primary B-cell lymphomas: From diagnosis to treatment. An Bras Dermatol. 2015;90(5):687-706.

8. Grange F, Beylot-Barry M, Courville $P$ et al. Primary cutaneous diffuse large B-cell lymphoma, leg type clinicopathologic features and prognostic analysis in 60 Cases. Arch. Dermatol. 2007;143(9):11441150.

9. Kim $\mathrm{YH}$, Willemze R, Pimpinelli $\mathrm{N}$ et al. TNM classification system for primary cutaneous lymphomas other than mycosis fungoides and Sezary syndrome: a proposal of the International Society for Cutaneous Lymphomas (ISCL) and the Cutaneous Lymphoma Task Force of the European Organization of Research and Treatment of Cancer (EORTC). Blood. 2007;110(2):479-484.

10. Oken MM, Creech RH, Tormey DC et al. Toxicity and response criteria of the eastern cooperative oncology group. Am. J. Clin. Oncol. 1982;5(6):649-655.
11. Kodama K, Massone C, Chott A et al. Primary cutaneous large B-cell lymphomas: clinicopathologic features, classification, and prognostic factors in a large series of patients, Blood. 2005;106(7):2491-2497.

12. Wilcox RA. Cutaneous B-cell lymphomas. 2016 update on diagnosis, riskstratification, and management. $\mathrm{Am} \mathrm{J}$ Hematol. 2016;91(10):1052-1055.

13. Zhao J, Han B, Shen T et al. Primary cutaneous diffuse large B-cell lymphoma (leg type) after renal allograft: case report and review of the literature. Int $J$ Hematol. 2009;89(1):113-117.

14. Nakayama-Ichivarna S, Yokote T, Iwaki K et al. Co-infection of human herpesvirus- 6 and human herpesvirus- 8 in primary cutaneous diffuse large B-cell lymphoma, leg type. $\mathrm{Br}$ J Haematol. 2011;155(4):514-516.

15. Sousa ARD, Costa IS, Araujo Filho EF et al. Primary cutaneous large B-cell lymphoma of atypical presentation: case report. An. Bras. Dermatol. 2011;86(3):549-551.

16. Massone C, Fink-Puches R, Wolf I, Atypical clinicopathologic presentation of primary cutaneous diffuse large B-cell lymphoma, leg type. J Am Acas Dermatol. 2015;72(6):1016-20.

17. Bancroft LW, Peterson JJ, Kransdorf MJ et al. Soft tissue tumors of the lower extremities. Radiol Clin North Am. 2002;40(5):991-1011.

18. Khan AJ, Usman Fm Abbasi S, et al. Diffuse large B-cell lymphoma presenting as a chronic leg ulcer: The importance of repeat tissue biopsy. Ann R Coll Surg Engl. 2011;93:e9-e10.

19. Süss A, Simon JC, Sticherling M. Primary cutaneous diffuse large B-cell lymphoma, leg type, with the clinical picture of chronic venous ulceration. Acta Derm Venereol. 2007;87:169-170.

20. Church DJ, Krumme J, Kotwal S. Evaluating Soft-Tissue Lumps and Bumps. Mo Med. 2017;114(4):289-294.

21. Hope CB, Pincus LB. Primary cutaneous $B$-cell lymphomas with large cell predominance-primary cutaneous follicle center lymphoma, diffuse large B-cell lymphoma, leg type and intravascular large B-cell lymphoma. Semin Diagn Pathol. 2017;34(1):85-98.

22. Lucioni M, Berti E, Arcaini L et al. Primary cutaneous B-cell lymphoma other than marginal zone: clinicopathologic analysis of 161 cases: comparison with current classification and definition of prognostic markers. Cancer Med. 2016;5(10):27402755.

23. Koens L, Vermer MH, Willemze R, et al. IgM expression on paraffin sections distinguishes primary cutaneous large B-cell lymphoma, leg type from primary cutaneous follicle center lymphoma. Am J Surg Pathol. 2010;34(7):1043-1048.

24. Demirkesen $C$, Tüzüner $N$, Esen $T$ et al. The expression of IgM is helpful in the differentiation of primary cutaneous diffuse large $B$ cell lymphoma and follicle center lymphoma. Leuk Res. 2011;35(9):12691272.

25. Senff NJ, Zoutman W, Vermeer MH et al. Fine-Mapping Chromosomal Loss at 9p21: Correlation with Prognosis in Primary Cutaneous Diffuse Large B-Cell Lymphoma, Leg Type. J Invest Dermatol. 2009;129(5):1149-1155.

26. Kim EJ, Lewis DJ, Duvic M. Novel Mutations Involving NF-KB and B-Cell Signaling Pathways in Primary Cutaneous Large B-Cell Lymphoma, Leg-Type and Comparison with Sézary Syndrome. J Invest Dermatol. 2017;137(9):1831-1833.

27. Pham-Ledard A, Prochazkova-Carlotti M, Andrique I et al. Multiple genetic alterations in primary cutaneous large B-cell lymphoma, leg type support a common lymphomagenesis with activated B-cell-like diffuse large B-cell lymphoma. Mod Pathol. 2014;27:402-411.

28. Mareshal S, Pham-Ledard A, Viailly PJ et al. Identification of somatic mutations in primary cutaneous diffuse large B-cell lymphoma, leg type by massive parallel sequencing. J Invest Dermatol. 2017;137(9):1984-1994.

29. Jardin F, Mareschal S, Pham-Ledard A et al. The landscape of somatic mutation of primary cutaneous diffuse large B-cell lymphoma, leg type. Hematol Oncol. 2017;35(S2):107-109.

30. Goodlad JR, van Hoppe S, Ahmed S et al. Molecular analysis of primary cutaneous diffuse large B-cell lymphoma, leg type. Hematol Oncol. 2017;35(S2):278-279.

31. Deng AL, Kim YR, Lichtenstein EA et al. Combination of ibrutinib and chemotherapy produced a durable remission in multiply relapsed diffuse large B-cell lymphoma leg type with mutant MYD88 and wild type CD79. Haematologica. 2017;102(7):e275-e277.

32. Lee JH, Jeong $\mathrm{H}$, Choi JW et al. Clinicopathologic significance of MYD88 
L265P mutation in diffuse large B-cell lymphoma: A meta-analysis. Sci Rep. 2017;7(1):1785.

33. Sokolowska-Wojdylo M, Olek-Hrab K, Ruckemann-Dziurdzinska K. Primary cutaneous lymphomas: diagnosis and treatment. Postepv Dermatol Alergol. 2015;32(5):368-383.

34. Gupta E, Accurso J, Sluzevich J et al. Excellent outcome of immunomodulation or bruton's tyrosine kinase inhibition in highly refractory primary cutaneous diffuse large B-cell lymphoma, leg type. Rare Tumors. 2015;7(4):6067.

35. Toberer F, Mechtersheimer G, Jaschinski H et al. Spontaneous regression of primary cutaneous diffuse large B-cell lymphoma, leg type. Acta Derm Venereol. 2018:98(6):608-609.
36. Jimura N, Fujii K, Baba A, et al. Spontaneous regression of a primary cutaneous diffuse large B-cell lymphoma, leg type. Letter to the Editor. The Journal of Dermatology. 2017;44(5):608-609.

37. Rao S, Morris R, Rice ZP et al. Regression of diffuse B-cell lymphoma of the leg with intralesional gentian violet. Exp Dermatol. 2018;27(1):93-95. 